$\mathbb{T}$ periodica polytechnica

Mechanical Engineering

$56 / 1(2012) 2731$

web: http://www.pp.bme.hu/me

(c) Periodica Polytechnica 2012

RESEARCH ARTICLE

\section{Analytical strain solution for the Drucker-Prager elastoplasticity model with linear isotropic hardening}

\author{
Attila Kossa
}

Received 2012-04-30

\begin{abstract}
This article presents the analytical strain solution of the rateform non-associative elastic-plastic constitutive model using the Drucker-Prager yield criterion with linear isotropic hardening. The strain solution is obtained using constant stress rate assumption. The solution for the deviatoric radial loading case is also presented. In addition, the strain solution for the case, when the stress state is located at the apex of the yield surface, is also derived.
\end{abstract}

\section{Keywords}

elastoplasticity;Drucker-Prager;linear hardening;analytical solution.

\section{Attila Kossa}

Department of Applied Mechanics, BME, H-1111 Budapest, Múegyetem rkp. 5 , Hungary

e-mail: kossa@mm.bme.hu

\section{Introduction}

The structure of a particular rate-form elastic-plastic constitutive equation strongly depends on the yield criterion used in the formulation. The Drucker-Prager yield criterion is a widely used criterion for materials, where the hydrostatic pressure affects the plastic deformation. Experimental results indicated that using non-associative flow rule with combination of the Drucker-Prager yield criterion gives a more accurate approach in the constitutive modelling. In addition, the elastoplastic constitutive model can be extended by including the hardening behavior of the material.

In the stress-driven case, the loading is given by a prescribed stress input and the corresponding strain response is sought. This strain solution can be obtained in a numerical way, but the importance of an analytical solution is obvious. Obtaining the analytical strain solution for a general stress input is usually very complicated or even impossible. If constant stress rate input is considered, then the governing equations have much simpler structure, which allows us to obtain the strain solution in an analytical way. For the associative von Mises elastoplasticity model with combined linear hardening rule, Kossa and Szabó [6] presented the exact strain solution applying the solution method proposed by Krieg and Krieg [7]. The analytical strain solution for the non-associative Drucker-Prager elastoplasticity model governed by linear isotropic hardening was presented by Szabó and Kossa [11]. The latter solution can be converted to a simpler form by utilizing the Kriegs' technique. This paper is devoted to present this novel strain solution.

Section 2 briefly summarizes the derivation of the constitutive equation for the non-associative Drucker-Prager elastoplastic model with linear isotropic hardening. Then, Section 3 presents the analytical solution method to obtain the corresponding strain response for constant stress rate input. Besides the general solution, the solutions for the deviatoric radial loading case and for the apex problem are also derived.

In order to simplify the presentation of the formulae, specific font styles are used to represent different mathematical quantities. The convention employed for this reason is the following: scalar-valued functions is denoted by italic light-face letters (e.g. 
$p, E, G)$; vectors and second-order tensors are denoted by italic bold-face letters (e.g. $s, \sigma, \varepsilon)$; fourth-order tensors are written as italic bold-face calligraphic letters (e.g. $\mathcal{T}, \mathcal{D}^{\mathrm{e}}$ ). Furthermore, the following operations are employed: $\operatorname{tr} \boldsymbol{A}$ means the trace of $\boldsymbol{A} ; \boldsymbol{A}^{T}$ denotes the transpose of $\boldsymbol{A} ; \dot{\boldsymbol{A}}$ indicates the material time derivative of $\boldsymbol{A} ; \boldsymbol{A}^{-1}$ denotes the inverse of $\boldsymbol{A} ;\|\boldsymbol{A}\|$ is the Euclidean norm of $\boldsymbol{A} ; \boldsymbol{A} \otimes \boldsymbol{B}$ represents the dyadic (or tensor) product of $\boldsymbol{A}$ and $\boldsymbol{B} ; \boldsymbol{A}: \boldsymbol{B}$ indicates the double dot product (or double contraction) between $\boldsymbol{A}$ and $\boldsymbol{B},\left(\boldsymbol{A}: \boldsymbol{B}=A_{i j} B_{i j}\right)$. Second-order and fourth-order identity tensors are denoted by $\delta$ and $\mathcal{I}$, respectively.

\section{Constitutive equation of the non-associative Drucker-Prager elastoplasticity model governed by linear isotropic hardening}

The Drucker-Prager yield criterion is a simple modification of the von Mises criterion, in which the hydrostatic stress component is also included to introduce pressure-sensitivity [4]. The yield function for this case can be written as [2, 5, 12]

$$
F\left(\sigma, \sigma_{Y}, \alpha\right)=\frac{1}{\sqrt{2}} S+3 \alpha p-k,
$$

where $S=\|\boldsymbol{s}\|, \boldsymbol{s}=\boldsymbol{\sigma}-p \boldsymbol{\delta}$ denotes the deviatoric stress, $p=$ $\operatorname{tr} \sigma / 3$ is the hydrostatic stress, $\sigma_{Y}$ represents the yield stress, $\alpha$ is a material parameter and $k$ is related to the yield stress as [1, 12]

$$
k\left(\bar{\varepsilon}^{p}\right)=\left(\alpha+\frac{1}{\sqrt{3}}\right) \sigma_{Y}\left(\bar{\varepsilon}^{p}\right) .
$$

In $(2), \bar{\varepsilon}^{p}$ denotes the accumulated plastic strain, which is defined by the relation [1]

$$
\bar{\varepsilon}^{p}=\sqrt{\frac{2}{3}} \int_{0}^{t}\left\|\dot{\boldsymbol{\varepsilon}}^{p}\right\| \mathrm{d} \tau
$$

The yield surface in the principal stress space is represented by a circular cone around the hydrostatic axis (see Fig. 1).

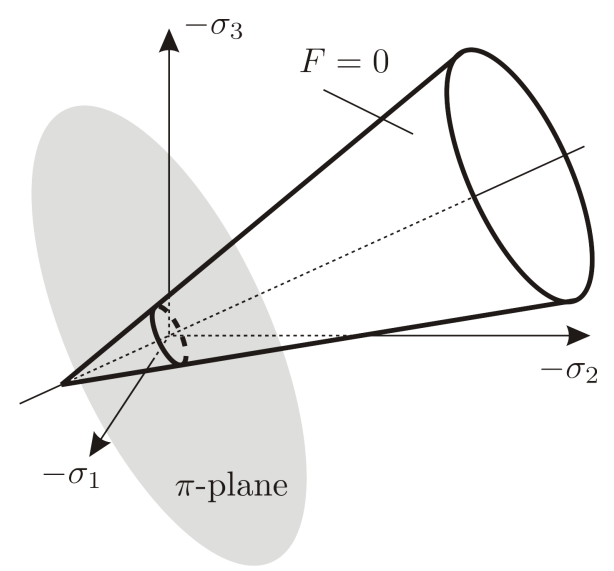

Fig. 1. Illustration of the Drucker-Prager yield surface in the principal stress space

The material starts to deform plastically, when the yield surface is reached. Upon further loading, the deformation produces plastic flow. The direction of the plastic strain rate is defined according to the non-associative plastic flow rule

$$
\dot{\boldsymbol{\varepsilon}}^{p}=\dot{\lambda} \frac{\partial g}{\partial \sigma}
$$

where the scalar function $\dot{\lambda}$ denotes the plastic multiplier, whereas $g$ is the plastic potential function, which itself is a function of the stresses. A commonly adopted form is given by [1]

$$
g=\frac{1}{\sqrt{2}} S+3 \beta p,
$$

where $\beta$ is an additional material parameter. The gradients of the yield function and the plastic potential function, with respect to $\sigma$ are the following:

$$
\boldsymbol{N}=\frac{\partial F}{\partial \boldsymbol{\sigma}}=\frac{\boldsymbol{s}}{\sqrt{2} S}+\alpha \boldsymbol{\delta}, \quad \boldsymbol{Q}=\frac{\partial g}{\partial \boldsymbol{\sigma}}=\frac{\boldsymbol{s}}{\sqrt{2} S}+\beta \boldsymbol{\delta} .
$$

If the material behavior, in the plastic region of the uniaxial stress-strain curve, is modelled with linear schematization, then we arrive at the linear isotropic hardening rule

$$
\sigma_{Y}\left(\bar{\varepsilon}^{p}\right)=\sigma_{Y 0}+H \bar{\varepsilon}^{p},
$$

where the slope of the curve is given by the constant plastic hardening modulus $H$.

The loading/unloading conditions can be expressed in the Kuhn-Tucker form as [2, 8, 10]

$$
\dot{\lambda} \geq 0, \quad F \leq 0, \quad \dot{\lambda} F=0 .
$$

The general form of the linear elastic stress-strain relation for isotropic material can be written as

$$
\sigma=\mathcal{D}^{\mathrm{e}}: \varepsilon
$$

where $\mathcal{D}^{\mathrm{e}}$ denotes the fourth-order elasticity tensor, which can be formulated as Doghri [3]

$$
\mathcal{D}^{\mathrm{e}}=2 G \mathcal{T}+K \boldsymbol{\delta} \otimes \boldsymbol{\delta}
$$

where $G$ stands for the shear modulus, $K$ denotes the bulk modulus, whereas $\mathcal{T}$ is the fourth-order deviatoric tensor, $\mathcal{T}=$ $\mathcal{I}-\frac{1}{3} \boldsymbol{\delta} \otimes \boldsymbol{\delta}$.

The plastic multiplier can be obtained from the consistency condition $\dot{F}=0$ :

$$
\begin{array}{r}
\dot{F}=\frac{\partial F}{\partial \boldsymbol{\sigma}}: \dot{\boldsymbol{\sigma}}-\dot{k}=\boldsymbol{N}: \mathcal{D}^{\mathrm{e}}: \dot{\boldsymbol{\varepsilon}}- \\
\dot{\lambda}\left(\boldsymbol{N}: \mathcal{D}^{\mathrm{e}}: \boldsymbol{Q}+H\left(\alpha+\frac{1}{\sqrt{3}}\right) \sqrt{\frac{1}{3}+2 \beta^{2}}\right),
\end{array}
$$

$$
\begin{aligned}
& \dot{\lambda}=\frac{\boldsymbol{N}: \mathcal{D}^{\mathrm{e}}: \dot{\boldsymbol{\varepsilon}}}{\boldsymbol{N}: \mathcal{D}^{\mathrm{e}}: \boldsymbol{Q}+H\left(\alpha+\frac{1}{\sqrt{3}}\right) \sqrt{\frac{1}{3}+2 \beta^{2}}}=
\end{aligned}
$$

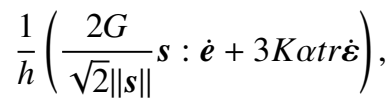


where the scalar parameter $h$ is defined as

$$
h=G+9 K \alpha \beta+j, \quad j=H\left(\alpha+\frac{1}{\sqrt{3}}\right) \sqrt{\frac{1}{3}+2 \beta^{2}} .
$$

The elastoplastic tangent tensor $\mathcal{D}^{\mathrm{ep}}$ is derived from the relation

$$
\begin{aligned}
\dot{\boldsymbol{\sigma}} & =\mathcal{D}^{\mathrm{e}}: \dot{\boldsymbol{\varepsilon}}^{e}=\mathcal{D}^{\mathrm{e}}: \dot{\boldsymbol{\varepsilon}}-\mathcal{D}^{\mathrm{e}}: \dot{\boldsymbol{\varepsilon}}^{p} \\
& =\mathcal{D}^{\mathrm{e}}: \dot{\boldsymbol{\varepsilon}}-\frac{\boldsymbol{N}: \mathcal{D}^{\mathrm{e}}: \dot{\boldsymbol{\varepsilon}}}{h} \mathcal{D}^{\mathrm{e}}: \boldsymbol{Q} \\
& =\left(\mathcal{D}^{\mathrm{e}}-\frac{\mathcal{D}^{\mathrm{e}}: \boldsymbol{Q} \otimes N: \mathcal{D}^{\mathrm{e}}}{h}\right): \dot{\boldsymbol{\varepsilon}} .
\end{aligned}
$$

Using the result above, the elastoplastic constitutive law can be written as

$$
\dot{\boldsymbol{\sigma}}=\mathcal{D} \dot{\boldsymbol{\varepsilon}}
$$

where

$$
\begin{aligned}
& \mathcal{D}^{\mathrm{ep}}=\mathcal{D}^{\mathrm{e}}-\frac{\mathcal{D}^{\mathrm{e}} \otimes \boldsymbol{Q} \otimes N: \mathcal{D}^{\mathrm{e}}}{h}= \\
& \mathcal{D}^{\mathrm{e}}-\frac{1}{h}\left(\frac{2 G^{2}}{S^{2}} \boldsymbol{s} \otimes s+\frac{6 K G \alpha}{\sqrt{2} S} \boldsymbol{s} \otimes \boldsymbol{\delta}+\right. \\
&\left.\frac{6 K G \beta}{\sqrt{2} S} \delta \otimes s+9 K^{2} \alpha \beta \delta \otimes \delta\right) .
\end{aligned}
$$

The constitutive equation 16 can be separated into deviatoric and hydrostatic parts as follows

$$
\dot{\boldsymbol{s}}=2 G \dot{\boldsymbol{e}}-\frac{2 G^{2}}{h S^{2}}\left(\boldsymbol{s}: \dot{\boldsymbol{e}}+\frac{3 K \alpha S \operatorname{tr} \dot{\boldsymbol{\varepsilon}}}{\sqrt{2} G}\right) \boldsymbol{s}
$$

and

$$
\dot{p}=K \operatorname{tr} \dot{\boldsymbol{\varepsilon}}-\frac{3 \sqrt{2} K G \beta}{h S}\left(s: \dot{\boldsymbol{e}}+\frac{3 K \alpha S \operatorname{tr} \dot{\varepsilon}}{\sqrt{2} G}\right) .
$$

\section{Inverse elastoplastic constitutive equation}

The inverse of the constitutive law $[16$ ) is defined as

$$
\dot{\boldsymbol{\varepsilon}}=\boldsymbol{C}^{\mathrm{ep}}: \dot{\boldsymbol{\sigma}}
$$

where the fourth-order elastoplastic compliance tangent tensor $C^{\text {ep }}$ is obtained by the inversion of 17 using the ShermanMorrison formula [9, 11]

$$
\begin{aligned}
\boldsymbol{C}^{\mathrm{ep}} & =\left(\mathcal{D}^{\mathrm{ep}}\right)^{-1}=C^{\mathrm{e}}+\frac{1}{j} \boldsymbol{Q} \otimes \boldsymbol{N} \\
& =\boldsymbol{C}^{\mathrm{e}}+\frac{1}{j}\left(\frac{1}{2 S^{2}} \boldsymbol{s} \otimes \boldsymbol{s}+\frac{\alpha}{\sqrt{2} S} \boldsymbol{s} \otimes \boldsymbol{\delta}+\right. \\
& \left.+\frac{\beta}{\sqrt{2} S} \boldsymbol{\delta} \otimes \boldsymbol{s}+\alpha \beta \boldsymbol{\delta} \otimes \boldsymbol{\delta}\right) .
\end{aligned}
$$

$C^{\mathrm{e}}$ denotes the fourth-order elastic compliance tensor, the inverse of $\mathcal{D}^{\mathrm{e}}[3]$ :

$$
C^{\mathrm{e}}=\frac{1}{2 G} \mathcal{I}-\frac{v}{E} \boldsymbol{\delta} \otimes \boldsymbol{\delta}=\frac{1}{2 G} \mathcal{T}+\frac{1}{9 K} \boldsymbol{\delta} \otimes \boldsymbol{\delta},
$$

where $v$ is the Poisson's ratio and $E$ is the Young's modulus.
The inverse constitutive law 21 can be separated into deviatoric and hydrostatic part as follows:

$$
\dot{\boldsymbol{e}}=\frac{1}{2 G} \dot{\boldsymbol{s}}+\frac{1}{2 j S^{2}}(\boldsymbol{s}: \dot{\boldsymbol{s}}+3 \sqrt{2} S \alpha \dot{p}) \boldsymbol{s}
$$

and

$$
\frac{1}{3} \operatorname{tr} \dot{\boldsymbol{\varepsilon}}=\left(\frac{1}{3 K}+\frac{3 \alpha \beta}{j}\right) \dot{p}+\frac{\beta(s: \dot{\boldsymbol{s}})}{\sqrt{2} j S}
$$

\section{Analytical strain solution for stress-driven case}

Under stress-driven formulation, it is assumed that the total and plastic strain fields, the stress field and the internal variables appearing in the particular model are known at an instant time $t_{n} \in[0, T]$, where $[0, T] \subset \mathbb{R}$ denotes the time interval under consideration. Furthermore, the stress field $\sigma$ is given in the whole interval $[0, T]$, consequently, the loading history is defined by the given stress field $\sigma(t)$. Therefore, in stress-driven problems, the strain field, the plastic strain field and the internal variables have to be determined for a given time $t \in\left[t_{n}, T\right]$, $t>t_{n}$.

In the following, the solution is derived for the case when $\dot{\sigma}$ is constant, thus

$$
\begin{gathered}
\sigma=\sigma_{n}+\dot{\boldsymbol{\sigma}}\left(t-t_{n}\right), \\
\boldsymbol{s}=\boldsymbol{s}_{n}+\dot{\boldsymbol{s}}\left(t-t_{n}\right), \\
p=p_{n}+\dot{p}\left(t-t_{n}\right) .
\end{gathered}
$$

For simplicity of the presentation the dependence on variable $t$ is omitted in the following expressions.

Define the angle $\omega$ through the following inner product:

$$
\boldsymbol{s}: \dot{\boldsymbol{s}}=S\|\dot{\boldsymbol{s}}\| \cos \omega
$$

The plastic multiplier, by combining (12, 25 and 26 then becomes

$$
\dot{\lambda}=\frac{\|\dot{\boldsymbol{s}}\|}{\sqrt{2} j}\left(\cos \omega+\frac{3 \sqrt{2} \alpha \dot{p}}{\|\dot{\boldsymbol{s}}\|}\right) .
$$

Thus, plastic loading occurs when

$$
\dot{p}>-\frac{\|\dot{s}\|}{3 \sqrt{2} \alpha} \cos \omega .
$$

From [28] it follows that

$$
\dot{S}=\|\dot{\boldsymbol{S}}\| \cos \omega .
$$

Taking the time derivative of 28 and then combining it with 31] gives

$$
\dot{\omega}=-\frac{\|\dot{s}\| \sin \omega}{S} .
$$

Dividing (31) with 32 yields the separable differential equation:

$$
\frac{1}{S} \mathrm{~d} S=-\frac{1}{\tan \omega} \mathrm{d} \omega
$$


with the initial condition $\omega\left(t=t_{n}\right)=\omega_{n}$ and $S\left(t=t_{n}\right)=S_{n}$. Thus the parameter $S$ can be written as a function of the angle $\omega$ :

$$
S=S_{n} \frac{\sin \omega_{n}}{\sin \omega}
$$

By substituting this solution back into expression (32) we arrive at the separable differential equation

$$
\frac{1}{\sin ^{2} \omega} \mathrm{d} \omega=-\frac{\|\dot{\boldsymbol{s}}\|}{S_{n} \sin \omega_{n}} \mathrm{~d} t
$$

which has the solution

$$
\omega=\arctan \left(\frac{S_{n} \sin \omega_{n}}{S_{n} \cos \omega_{n}+\|\dot{\boldsymbol{s}}\|\left(t-t_{n}\right)}\right) .
$$

Combining 27, 236 and 25 we can express $s$ in terms of the angle $\omega$ as

$$
\boldsymbol{s}=\boldsymbol{s}_{n}+\frac{S_{n}}{\|\dot{\boldsymbol{s}}\|} \frac{\sin \left(\omega_{n}-\omega\right)}{\sin \omega} \dot{\boldsymbol{s}} .
$$

Inserting (37) and 34) into 25, the deviatoric strain rate can be written in the form

$$
\begin{aligned}
\dot{\boldsymbol{e}}=\frac{1}{2 G} \dot{\boldsymbol{s}}+ & \frac{\|\dot{\boldsymbol{s}}\| \cos \omega \sin \omega}{2 S_{n} j \sin \omega_{n}} \boldsymbol{s}_{n}+\frac{1}{2 j} \frac{\sin \left(\omega_{n}-\omega\right) \cos \omega}{\sin \omega_{n}} \dot{\boldsymbol{s}} \\
& +\frac{\alpha \operatorname{tr} \dot{\boldsymbol{\sigma}} \sin \omega}{\sqrt{2} j S_{n} \sin \omega_{n}} \boldsymbol{s}_{n}+\frac{\alpha \operatorname{tr} \dot{\boldsymbol{\sigma}}}{\sqrt{2} j\|\dot{\boldsymbol{s}}\|} \frac{\sin \left(\omega_{n}-\omega\right)}{\sin \omega_{n}} \dot{\boldsymbol{s}} .
\end{aligned}
$$

Integrating both sides, using 35, yields the solution for the deviatoric strain as

$$
\boldsymbol{e}=\boldsymbol{e}_{n}+A \boldsymbol{s}_{n}+B \dot{\boldsymbol{s}}
$$

where the parameters $A_{e}$ and $B_{e}$, after simplification, become

$$
\begin{aligned}
& A=\frac{1}{2 j} \ln \left(\frac{S}{S_{n}}\right)+\frac{\alpha \operatorname{tr} \dot{\boldsymbol{\sigma}}}{\sqrt{2} j\|\dot{\boldsymbol{s}}\|} \ln \left(\frac{\tan \frac{\omega_{n}}{2}}{\tan \frac{\omega}{2}}\right), \\
& B=\frac{\left(t-t_{n}\right)}{2}\left(\frac{1}{G}+\frac{1}{j}\right)+\frac{\alpha \operatorname{tr} \dot{\boldsymbol{\sigma}}\left(S-S_{n}\right)}{\sqrt{2} j\|\dot{\boldsymbol{s}}\|^{2}}- \\
& \frac{S_{n} \sin \omega_{n}}{\|\dot{\boldsymbol{s}}\|}\left(\frac{A}{\tan \omega_{n}}+\frac{\omega_{n}-\omega}{2 j}\right) .
\end{aligned}
$$

Inserting 28) and (31) into expression 26 results in the differential equation

$$
\frac{1}{3} \operatorname{tr} \dot{\varepsilon}=\left(\frac{1}{3 K}+\frac{3 \alpha \beta}{j}\right) \dot{p}+\frac{\beta}{\sqrt{2} j} \dot{S},
$$

which can be simply integrated yielding the solution

$$
\frac{1}{3} \operatorname{tr} \varepsilon=\frac{1}{3} \operatorname{tr} \varepsilon_{n}+\left(\frac{1}{3 K}+\frac{3 \alpha \beta}{j}\right) \dot{p}\left(t-t_{n}\right)+\frac{\beta\left(S-S_{n}\right)}{\sqrt{2} j} .
$$

Finally, the total strain solution is computed by combining 39 and 43 .

\section{Solution in the case of deviatoric radial loading}

The solution reduces to simpler form in the case when $\omega_{n}=$ 0 or $\omega_{n}=\pi$ or $\|\dot{s}\|=0$. These scenarios correspond to the deviatoric radial loading case, when we can write that

$$
S=S_{n}+q\|\dot{s}\|\left(t-t_{n}\right), \quad s=\frac{S}{S_{n}} \boldsymbol{s}_{n},
$$

where $q$ is defined by

$$
q=\left\{\begin{aligned}
1 & \text { if } \omega_{n}=0 \\
-1 & \text { if } \omega_{n}=\pi \\
0 & \text { if }\|\dot{\boldsymbol{s}}\|=0
\end{aligned}\right.
$$

Inserting (44) into 25 and 26 gives

$$
\begin{gathered}
\dot{\boldsymbol{e}}=\frac{1}{2 G} \dot{\boldsymbol{s}}+\frac{3 \alpha \dot{p}}{\sqrt{2} S_{n} j} \boldsymbol{s}_{n}+q \frac{1}{2 j} \dot{\boldsymbol{s}}, \\
\frac{1}{3} \operatorname{tr} \dot{\boldsymbol{\varepsilon}}=\left(\frac{1}{3 K}+\frac{3 \alpha \beta}{j}\right) \dot{p}+q \frac{\beta\|\dot{\boldsymbol{s}}\|}{\sqrt{2} j} .
\end{gathered}
$$

Thus, the solutions for the strains will be

$$
\begin{gathered}
\boldsymbol{e}=\boldsymbol{e}_{n}+\frac{3 \alpha \dot{p}\left(t-t_{n}\right)}{\sqrt{2} S_{n} j} \boldsymbol{s}_{n}+\left(\frac{1}{2 G}+q \frac{1}{2 j}\right)\left(t-t_{n}\right) \dot{\boldsymbol{s}}, \\
\frac{1}{3} \operatorname{tr} \boldsymbol{\varepsilon}=\frac{1}{3} \operatorname{tr} \boldsymbol{\varepsilon}_{n}+\left(\frac{1}{3 K}+\frac{3 \alpha \beta}{j}\right) \dot{p}\left(t-t_{n}\right)+q \frac{\beta\|\dot{\boldsymbol{s}}\|}{\sqrt{2} j}\left(t-t_{n}\right) .
\end{gathered}
$$

\section{Stress input required to reach the apex}

It is obviously follows that the apex can be reached only due to deviatoric radial loading. Denote $c_{a} \dot{\boldsymbol{\sigma}}\left(t-t_{n}\right)$ the stress input required to reach the apex. From 27) it is clearly follows that

$$
c_{a}=\frac{S_{n}}{\|\dot{\boldsymbol{s}}\|\left(t-t_{n}\right)} .
$$

\section{Solution at the apex}

When the initial stress state is located at the apex, then it is obvious that the new stress state leaves the apex when $\|\dot{\boldsymbol{s}}\|>0$. From 29 , it follows that plastic loading initiates if

$$
\|\dot{\boldsymbol{s}}\|>-3 \sqrt{2} \alpha \dot{p}
$$

otherwise elastic unloading occurs. The solutions (48) and 49] can be used by inserting $q=1$ into these expressions yielding

$$
\boldsymbol{e}=\boldsymbol{e}_{n}+\left(\frac{1}{2 G}+\frac{1}{2 j}\right)\left(t-t_{n}\right) \dot{\boldsymbol{s}}
$$

$$
\frac{1}{3} \operatorname{tr} \varepsilon=\frac{1}{3} \operatorname{tr} \varepsilon_{n}+\left(\frac{1}{3 K}+\frac{3 \alpha \beta}{j}\right) \dot{p}\left(t-t_{n}\right)+\frac{\beta\| \pm \dot{\boldsymbol{s}}\|}{\sqrt{2} j}\left(t-t_{n}\right) .
$$




\section{Conclusion}

This paper presented a novel analytical strain solution for the non-associative Drucker-Prager elastoplasticity model with linear isotropic hardening. The solution is valid under constant stress rate assumption, which can be represented as a linear stress path input in the stress space. The analytical strain solutions were obtained for the deviatoric radial loading case, and for the special scenario, when the initial stress state is located in the apex of the yield surface. The new solutions can be used to obtain reference solution for particular elastoplastic problems.

\section{Acknowledgement}

This research has been supported by the Hungarian Scientific Research Fund, Hungary (under Contract: OTKA, K72572). This support is gratefully acknowledged. This work is connected to the scientific program of the "Development of qualityoriented and harmonized $\mathrm{R}+\mathrm{D}+\mathrm{I}$ strategy and functional model at BME" project. This project is supported by the New Széchenyi Plan (Project ID: TÁMOP-4.2.1/B-09/1/KMR-20100002).

\section{References}

1 Chen W., Han D., Plasticity for Structural Engineers, J. Ross Publishing, New York, 2007.

2 de Souza Neto E., Perić D, Owen D., Computational methods for plasticity, Wiley, 2008.

3 Doghri I, Mechanics of deformable solids, Springer, 2000.

4 Drucker D., Prager W, Soil mechanics and plastic analysis or limit design, Quarterly of Applied Mathematics 10 (1952), 157-162.

5 Jirásek M, Inelastic analysis of structures, Wiley, England, 2002.

6 Kossa A, Szabó L, Exact integration of the von Mises elastoplasticity model with combined linear isotropic-kinematic hardening, International Journal of Plasticity 25 (2009), 1083-1106.

7 Krieg R., Krieg D., Accuracies of numerical solution methods for the elasticperfectly plastic model, Journal of Pressure Vessel Technolgy 99 (1977), 510-515, DOI 10.1115/1.3454568.

8 Luenberger D., Ye Y, Linear and nonlinear programming, 3rd, Springer, 2008.

9 Sherman J, Morrison W., Adjusment of an inverse matrix corresponding to a change in one element of a given matrix, The Annals of Mathematical Statistics 21 (1949), 124-127.

10 Simo J., Hughes T., Computational Inelasticity, Springer, New York, 1998.

11 Szabó L, Evaluation of elasto-viscoplastic tangent matrices without numerical inversion, Computers and Structures 21 (1985), 1235-1236, DOI 10.1016/0045-7949(85)90177-4.

12 Szabó L, Kossa A, A new exact integration method for the DruckerPrager elastoplastic model with linear isotropic hardening, International Journal of Solids and Structures 49 (2012), 170-190, DOI 10.1016/j.ijsolstr.2011.09.021. 